\title{
Detector Arrays for the James Webb Space Telescope Near-Infrared Spectrograph
}

Bernard J. Rauscher ${ }^{a}$, David Alexander ${ }^{a}$, Clifford K. Brambora ${ }^{a}$, Rebecca Derro ${ }^{a}$, Chuck Engler $^{a}$, Ori Fox ${ }^{a, b}$, Matthew B. Garrison ${ }^{a}$, Greg Henegar ${ }^{a}$, Robert J. Hill ${ }^{a, c}$, Thomas Johnson $^{a}$, Don J. Lindler ${ }^{p}$, Sridhar S. Manthripragada ${ }^{a}$, Cheryl Marshall ${ }^{a}$, Brent Mott $^{a}$, Thomas M. Parr ${ }^{a}$, Wayne D. Roher ${ }^{a, d}$, Kamdin B. Shakoorzadeh ${ }^{a, e}$, Miles Smith ${ }^{a}$, Augustyn Waczynski ${ }^{a, f}$, Yiting Wen ${ }^{a, g}$, Donna Wilson ${ }^{a}$, Wei Xia-Serafino ${ }^{a, f}$, Craig Cabelli ${ }^{h}$, Edward Cheng ${ }^{h, c}$, James Garnett ${ }^{h}$, Markus Loose ${ }^{h}$, Majid Zandian ${ }^{h}$, Joseph Zino $^{h}$, Timothy Ellis ${ }^{i}$, Bryan Howe ${ }^{i}$, Miriam Jurado ${ }^{i}$, Ginn Lee $^{i}$, John Nieznanski ${ }^{i}$, Peter Wallis ${ }^{i}$, James York ${ }^{i}$, Michael W. Regan ${ }^{j}$, Torsten Böker ${ }^{k}$, Guido De Marchi ${ }^{k}$, Pierre Ferruit ${ }^{l, m, n}$, Peter Jakobsen ${ }^{k}$, and Paolo Strada ${ }^{k}$

${ }^{a}$ NASA Goddard Space Flight Center, Greenbelt, MD, U.S.A.; ${ }^{b}$ Department of Astronomy, University of Virginia, P.O. Box 4000325, Charlottesville, VA, 22904, U.S.A. ${ }^{c}$ Conceptual

Analytics LLC, 8209 Woburn Abbey Road, Glenn Dale, MD, 20769, U.S.A.; ${ }^{d}$ Northrop Grumman Technical Services, 4276 Forbes Blvd., Lanham, MD, 20706, U.S.A.; ${ }^{e}$ AK Aerospace Technology Corp., 12970 Brighton Dam Rd, Clarksville, MD, 21029, U.S.A.; ${ }^{f}$ Global Science \&

Technologies, Inc., 7855 Walker Drive, Suite 200, Greenbelt, MD, 20770, U.S.A.; ${ }^{9}$ Muniz Engineering Inc., 7404 Executive Place, Suite 500, Lanham, MD, 20706, U.S.A.; ${ }^{h}$ Teledyne Imaging Sensors, 5212 Verdugo Way, Camarillo, CA, 93012, U.S.A.; ${ }^{i}$ ITT Space Systems Division, 1447 St. Paul Street, Rochester, NY, 14653, U.S.A.; ${ }^{j}$ Space Telescope Science Institute, 3700 San Martin Drive, Baltimore, MD, 21218, U.S.A.; ${ }^{k}$ ESTEC, Astrophysics Division, Postbus 299, Noordwijk, NL2200 AG, Netherlands ${ }^{l}$ Université de Lyon, Lyon, F-69003, France; ${ }^{m}$ Université Lyon 1, Observatoire de Lyon, 9 avenue Charles André, Saint-Genis Laval, F-69230, France; ${ }^{n} \mathrm{CNRS}$, UMR 5574, Centre de Recherche Astrophysique de Lyon; Ecole Normale Supérieure de Lyon, Lyon, F-69007, France; ${ }^{p}$ Sigma Space Corporation, 4801 Forbes Blvd., Lanham, MD, 20706, U.S.A.

\begin{abstract}
The James Webb Space Telescope's ( JWST) Near Infrared Spectrograph (NIRSpec) incorporates two $5 \mu \mathrm{m}$ cutoff $\left(\lambda_{c o}=5 \mu \mathrm{m}\right) 2048 \times 2048$ pixel Teledyne HgCdTe HAWAII-2RG sensor chip assemblies. These detector arrays, and the two Teledyne SIDECAR application specific integrated circuits that control them, are operated in space at $\mathrm{T} \sim 37 \mathrm{~K}$. In this article, we provide a brief introduction to NIRSpec, its detector subsystem (DS), detector readout in the space radiation environment, and present a snapshot of the developmental status of the NIRSpec DS as integration and testing of the engineering test unit begins.
\end{abstract}

Keywords: JWST, NIRSpec, Detectors, HgCdTe, HAWAII-2RG, H2RG

\section{INTRODUCTION}

The James Webb Space Telescope (JWST) was conceived as the scientific successor to the Hubble and Spitzer space telescopes. Of all JWST "near-infrared" $(\lambda=0.6-5 \mu \mathrm{m})$ instruments, the Near Infrared Spectrograph (NIRSpec) has the most demanding detector requirements. A team headquartered at NASA Goddard Space Flight Center (GSFC) is leading development of the NIRSpec Detector Subsystem (DS). The flight hardware

Further author information: (Send correspondence to B. J. Rauscher)

B. J. Rauscher: E-mail: Bernard.J.Rauscher@nasa.gov. Telephone: +1 301 286-4871 
is being built now by GSFC, Teledyne Imaging Systems (TIS) of Camarillo, CA, and ITT of Rochester, NY. Although launch, no earlier than 2013, is still some time away, the flight and flight spare units are scheduled for delivery to the European Space Agency in September and December, 2008, respectively. In this article, we focus on the developmental status of the DS as Integration and Test begins in August, 2007.

This article is structured as follows. In Section 2, we summarize JWST, NIRSpec, and the DS for those who may not already be familiar with them. This section includes a discussion of the DS requirements, which are arguably the most challenging JWST detector requirements. We have tried to keep this introductory discussion brief, and refer freely to more complete descriptions in the literature. In Section 3 we describe the current programatic status of the DS, with a focus on near term schedule and major deliveries. Section 4 is primarily a short description of recent test results using engineering grade SIDECAR ASICs to drive engineering grade HAWAII-2RG (H2RG) SCAs in GSFC's Detector Characterization Laboratory (DCL). The DCL will be used for characterization of the integrated DS before delivery to ESA. A companion paper by Mott et al. (2007) describes technical progress in the DCL in more detail. ${ }^{1}$ Finally, in Section 5, we describe how we plan to operate NIRSpec's detectors in the JWST radiation environment, and how noise scales with the number of samples in the baseline MULTIACCUM readout mode.

\section{THE JAMES WEBB SPACE TELESCOPE}

$J W S T$ is a large, cold, infrared-optimized space telescope designed to enable fundamental breakthroughs in our understanding of the formation and evolution of galaxies, stars, and planetary systems. The project is led by the United States National Aeronautics and Space Administration (NASA), with major contributions from the European and Canadian Space Agencies (ESA and CSA respectively). JWST will have an approximately 6.6-m diameter aperture, be passively cooled to below $\mathrm{T}=50 \mathrm{~K}$, and carry four scientific instruments: NIRSpec, a NIR Camera (NIRCam), a NIR Tunable Filter Imager (TFI), and a Mid-Infrared Instrument (MIRI). All four scientific instruments are located in the Integrated Science Instruments Module (ISIM), which lies in the focal plane behind the primary mirror. JWST is planned for launch early in the next decade on an Ariane 5 rocket to a deep space orbit around the Sun-Earth Lagrange point L2, about $1.5 \times 10^{6} \mathrm{~km}$ from Earth. The spacecraft will carry enough fuel for a 10-year mission.

JWST's scientific objectives fall into four broad themes. These are as follows; (1) The End of the Dark Ages, First Light and Re-ionization, (2) The Assembly of Galaxies, (3) The Birth of Stars and Protoplanetary Systems, and (4) Planetary Systems and the Origins of Life. Most NIR programs will require long, staring observations, limited by the zodiacal background at L2 in the case of NIRCam and the TFI, or by detector noise in the case of NIRSpec. For all of $J W S T^{\text {ns }}$ NIR instruments, modest $\approx 100-200 \mathrm{kHz}$ pixel rates will be the rule, with total observing times per target typically $>10^{4}$ seconds. TIS H2RGs have been selected as the detectors for all three $J W S T$ NIR instruments. For a more thorough overview of JWST, we refer the interested reader to Ref. 2 .

\subsection{Near Infrared Spectrograph}

NIRSpec (Fig. 1) will be the first slit-based astronomical multi-object spectrograph (MOS) to fly in space, and is designed to provide NIR spectra of faint objects at spectral resolutions of $R=100, R=1000$ and $R=2700$. The instrument's all-reflective wide-field optics, together with its novel microelectromechanical systems (MEMS) programmable micro-shutter array slit selection device and H2RG detector arrays, combine to allow simultaneous observations of $>100$ objects within a $3.5 \times 3.4$ arcmin field of view with unprecedented sensitivity. A selectable $3 \times 3$ arcsec Integral Field Unit (IFU) and five fixed slits are also available for detailed spectroscopic studies of single objects. NIRSpec is presently expected to be capable of reaching a continuum flux of $20 \mathrm{nJy}(\mathrm{AB}>28)$ in $\mathrm{R}=100$ mode, and a line flux of $6 \times 10^{-19} \mathrm{erg} \mathrm{s}^{-1} \mathrm{~cm}^{-2}$ in $\mathrm{R}=1000$ mode at $\mathrm{S} / \mathrm{N}>3$ in $10^{4} \mathrm{~s}$.

NIRSpec is being built for the ESA by EADS Astrium as part of ESA's contribution to the JWST mission. The NIRSpec micro-shutter and detector arrays are provided by NASA/GSFC. 


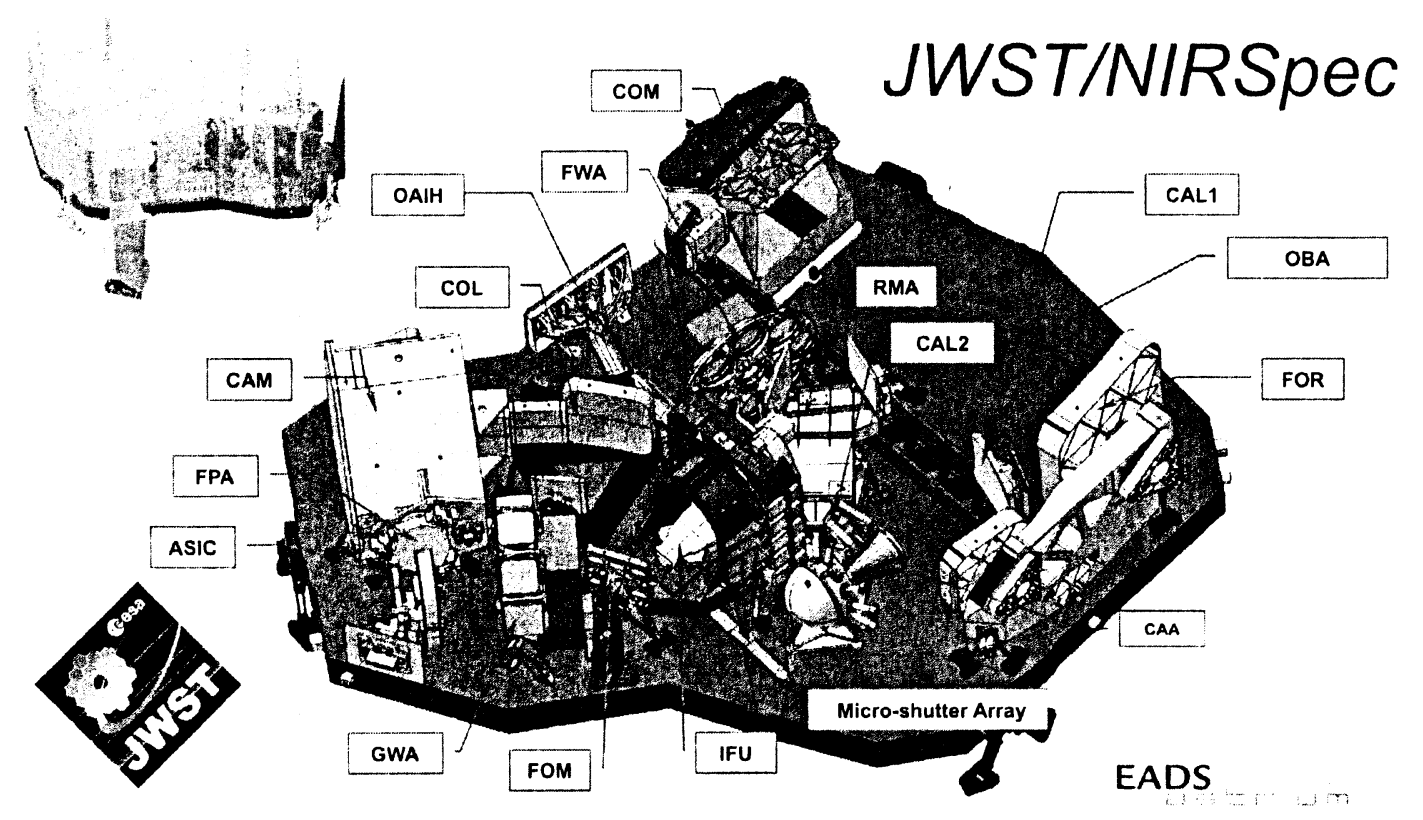

Figure 1. NIRSpec is being built by EADS/Astrium for the European Space Agency. NASA is providing the microelectromechanical systems (MEMS) micro-shutter array (MSA) for target selection and detector subsystem (DS), which is the focus of this paper. DS components that are visible in this figure include the focal plane assembly (FPA), two SIDECAR ASICs (labeled "ASIC" in the figure) for FPA control, and the cables connecting the ASICs to the FPA. Light enters the instrument from a pickoff mirror located below the element labeled "COM", passing through an opening in the optical bench assembly (OBA). For more information on the NIRSpec instrument, see the companion paper by Bagnasco et al. (2007) in these conference proceedings. ${ }^{3}$

\subsection{Detector Subsystem}

All three NIRSpec modes (MOS, IFU and fixed slits) share the need for large-format, high detective quantum efficiency (DQE), and ultra-low noise detectors covering the $\lambda=0.6-5 \mu \mathrm{m}$ spectral range. This need is fulfilled by two $\lambda_{\mathrm{co}}=5 \mu \mathrm{m}$ H2RG sensor chip assemblies (SCA). These SCAs, and the two TIS SIDECAR* application specific integrated circuits (ASICs) that will control them, represent today's state-of-the-art. This hardware is being delivered to the ESA by the NIRSpec DS team at GSFC. The DS team will deliver a fully integrated, tested, and characterized DS to ESA for integration into NIRSpec.

The DS (Figs. 2-3) consists of the following components; focal plane assembly (FPA), two SIDECAR ASICs, focal plane electronics (FPE), thermal and electrical harnesses, and software. The molybdenum FPA is being built by TIS and their partner ITT. The two H2RG SCAs are being built by TIS on packages supplied by ITT.

Figure 3 shows a block diagram of the DS. Light enters by striking the first surface of the FPA. Because NIRSpec is a wide-field MOS, the beam entering the FPA is a relatively fast $\sim f / 5.7$, with high incidence angles at the corners of the field. The fast beam drives NIRSpec's challenging $75 \mu \mathrm{m}$ peak-to-valley focal plane flatness requirement. Meeting this specification requires good control of TIS's SCA fabrication process and ITT-proprietary positioning and shimming techniques when installing SCAs into the FPA.

The SCA bias generators, clock drivers, timing control, and analog to digital converters are located in the SIDECAR ASICs. Each SCA has a dedicated SIDECAR. Both the SIDECARs and SCAs are operated at $\mathrm{T} \sim 37 \mathrm{~K}$

*SIDEC'AR: System for Image Digitization, Euhancement, Control and Retrieval. 

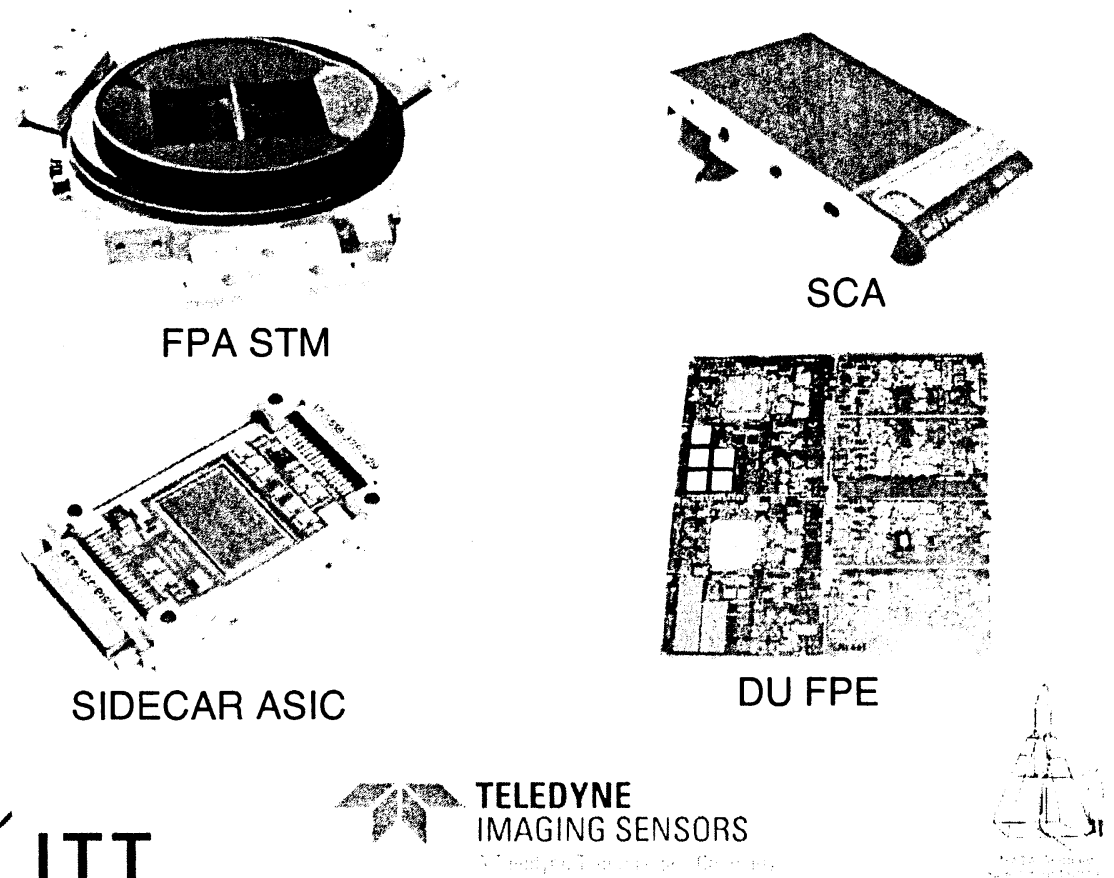

Figure 2. NASA is providing NIRSpec's DS. DS components include the focal plane assembly (FPA). Here we show the structural thermal model (STM) during test at ITT. The FPA contains two TIS HAWAII-2RG SCAs. Other components include two SIDECAR ASICs for FPA control and the focal plane electronics (FPE), which control the SIDECARs. This figure shows a development unit (DU) of the FPE undergoing test at NASA GSFC.

In NIR.Spec, the four outputs per SCA will appear as thick, $512 \times 2048$ pixels bands aligned with the dispersion direction. This is done to minimize the possibility of calibration difficulties in spectra that would otherwise span multiple outputs. Raw data will be averaged in the on-board focal plane array processor (FPAP) before being saved to the solid state recorder, and ultimately downlinked to the ground. The FPAP is located in the shared integrated command and data handling system (ICDH), and is not part of the DS. Averaging is done to conserve bandwidth for the data link to the ground. Following averaging, the data are still sampled-up-the-ramp, however each up-the-ramp data point has lower noise and the ramp is more sparsely sampled. For more information on detector readout, the interested reader is referred to Ref. 4.

\subsection{DS Requirements}

The top-level DS performance requirements are summarized in Table 1. These performance requirements ultimately flow from JWSTs 4 science themes (see Section 2). Broadly speaking the 4 science themes place a premium on sensitivity, photometric stability, and recovering quickly from saturation when observing bright sources.

In addition to the requirements listed in Table 1, the DS carries other requirements that pertain to operation within the NIRSpec instrument and/or the space environment at L2. These non-performance requirements are not a focus of this article, and will be discussed only when necessary to further some other aspect of the discourse.

While many readers may have a general knowledge of the JWST mission, we anticipate that few will have insight into the details of the sub-systems. In a departure from some NASA missions which take a conservative approach to minimize risk, many of $J W S T$ s sub-systems advance the state-of-the art. This is particularly true in the case of the NIRSpec DS. With demanding performance requirements including total noise $\sigma_{\text {total }} \leq 6 e^{-}$rms per $t_{\text {int }}$ integration, and dark current $i_{\text {dark }} \leq 0.010 e^{-} s^{-1}$ pixel $^{-1}$. NIRSpec's detector arrays will be among the most sensitive in existence. 


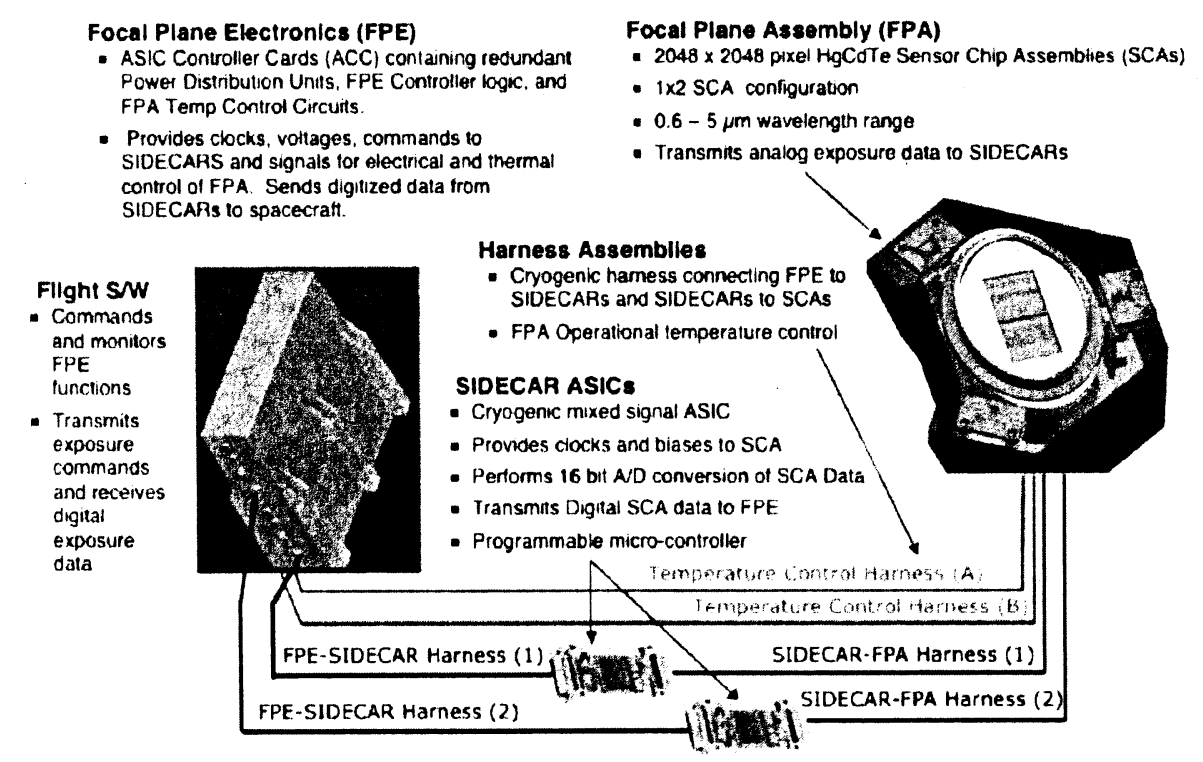

Figure 3. Block diagram of the NIRSpec DS. Light enters the FPA, which contains two identical anti-reflection coated H2RG SCAs. Each SCA has a dedicated SIDECAR ASIC for bias and clock generation and analog to digital conversion. The ASICs are connected to the SCAs by an approximately $600 \mathrm{~mm}$ long flat cable. Apart from one reference voltage that is generated by the FPE, the system is entirely digital downstream of the ASICs. The nearly all-digital nature of the system from this point on greatly mitigates the risk of electromagnetic interference (EMI) and electromagnetic compatibility (EMC) issues. The ASICs are connected to the T $\sim 300 \mathrm{~K} \mathrm{FPE}$ by another flat cable. The ASIC to FPE cable is about $4-\mathrm{m}$ long.

Table 1. Selected NIRSpec Detector Subsystem Performance Requirements

\begin{tabular}{|c|c|c|}
\hline Parameter & Requirement & Comment \\
\hline Total noise $\left(e^{-} \mathrm{rms}\right)$ & 6 & $\begin{array}{l}t_{\text {int }}=1008 \mathrm{~s} \\
\text { multi- } 22 \times 4 \text { sampling }\end{array}$ \\
\hline Mean dark current $\left(e^{-} s^{-1}\right.$ pixel $\left.^{-1}\right)$ & 0.010 & \\
\hline DQE & $\begin{array}{l}70 \% \\
80 \%\end{array}$ & $\begin{array}{l}0.6 \leq \lambda<1.0 \mu \mathrm{m} \\
1 \leq \lambda<5 \mu \mathrm{m}\end{array}$ \\
\hline Operating temperature (K) & $34-37$ & \\
\hline Pixel operability for science ${ }^{a}$ & $>92 \%$ & \\
\hline Persistence & $0.1 \%$ & $\begin{array}{l}\text { After a maximum of } 3 \text { de- } \\
\text { structive resets following ex- } \\
\text { posure to full well }\end{array}$ \\
\hline Well depth & $6 \times 10^{4} e^{-}$ & \\
\hline
\end{tabular}

${ }^{a}$ Pixel operability for science includes stringent thresholds on total noise and DQE. Pixels that fail to meet the operability for science requirement are degraded, although they may still be useful for target acquisition and other less sensitive observations. 
NIRSpec's exquisitely sensitive detector arrays were enabled by an aggressive JWST detector technology development program. Within the JWST Project, the detector development program is known by the name of the final NASA contract awarded, "Packaging and Manufacturing Technology (PMT)." By late 2003, the PMT program had produced two H2RGs that met the challenging dark current and total noise requirements. These were PMT parts H2RG-006-5.0 $\mu \mathrm{m}$ and H2RG-15-5.0 $\mu \mathrm{m}$. More recently, TIS has produced parts for JWST under the FGS/TFI flight program, notably H2RG-F007, that appear to be superior to the best PMT era parts.

Along the way to developing the H2RG SCA, JWST development produced a number of pathfinder parts that, were each the state-of-the-art when they were introduced. These included the $1 \mathrm{~K} \times 1 \mathrm{~K}$ pixels HAWAII-1R, which which introduced reference pixels, and the HAWAII-1RG, which added a programable "guide window." In parallel, NASA's Wide Field Camera 3 (WFC3) for the Hubble Space Telescope (HST) pioneered the use of $\lambda_{\text {co }}=1.7 \mu \mathrm{m} \mathrm{HgCdTe}$ detectors, which enabled ultra-low background operation at temperatures warmer than had hitherto been possible with TIS HgCdTe detector arrays.

\section{DS PROGRAMMATIC STATUS \& SCHEDULE}

The NIRSpec DS engineering test unit (ETU) is entering integration \& test (I\&T) now. Within NASA, an ETU is typically a high-fidelity representation of the actual flight hardware that is used for form, fit, and environmental qualification. The ETU is usually also used as the Qualification Unit.

In the September/October 2007 timeframe, electromagnetic interference and electromagnetic compatibility (EMI/EMC) testing of the ETU DS will begin at GSFC. EMI/EMC testing will be done with the FPA and SIDECAR ASICs at their $\mathrm{T} \sim 37 \mathrm{~K}$ operating temperature. The purpose of EMI/EMC testing is to surface any potential compatibility issues between JWST instruments. EMI/EMC testing will also provide the first opportunity to examine the integrated DS for noise pickup.

With regard to the flight module (FM) and flight spare(FS) units, TIS is is working to produce the flight SCAs and SIDECAR ASICs now, and fabrication of the FM FPA structure has begun at ITT.

\subsection{Detector Subsystem Schedule}

NASA is scheduled to deliver the ETU DS to ESA later this year (2007). The flight module (FM) is scheduled for delivery in September 2008, and the flight spare (FS) delivery is scheduled for December, 2008. These dates are all well before JWST launch, which is no earlier than June, 2013. The time between NASA's delivery of the DS to ESA and launch will be used for DS integration into NIRSpec, NIRSpec integration into the Integrated Science Instruments Module (ISIM), and ISIM integration into the observatory. Each of these higher levels of integration includes a test phase.

Significant near-term reviews include the FPA Critical Design Review (CDR) in Autumn, 2007, and the DS CDR in December 2007.

\section{DS TECHNICAL STATUS}

The NIRSpec DS is entering integration and test (I\&T) now. The most sensitive DS level tests in the I\&T flow will be done in NASA GSFC's DCL.

\subsection{NASA Goddard Space Flight Center Detector Characterization Laboratory}

The DCL will be used for integration and system level testing of the DS. The DCL is making steady progress toward a test readiness review in January, 2008. Specific progress includes the demonstration of ultra-low background detector operation in the "purple dewar", $i_{\text {dark }} \leq 0.010 e^{-} s^{-1}$ pixel $^{-1}$, and read noise, $\sigma_{\text {rcad }} \leq$ $19 e^{-}$rms. These tests are described in more detail in the companion paper by Mott et. al. (2007). which can be found in these conference proceedings. ${ }^{1}$

One significant recent accomplishment is the operation of NIRSpec H2RG SCAs using SIDECAR ASICs at $\mathrm{T} \sim 37 \mathrm{~K}$. The DCLs focal plane proxy and SIDECAR ASICs are shown in Figure 4. Although both the SIDECAR. A64, and SCA, H2RG-S024. are both engineering grade, the initial test results have nonetheless been impressive. 


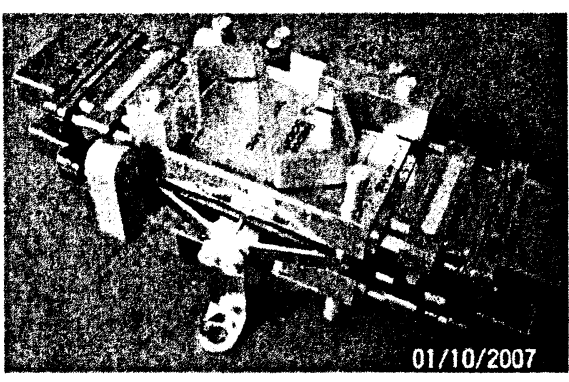

(a) SIDECAR ASICs

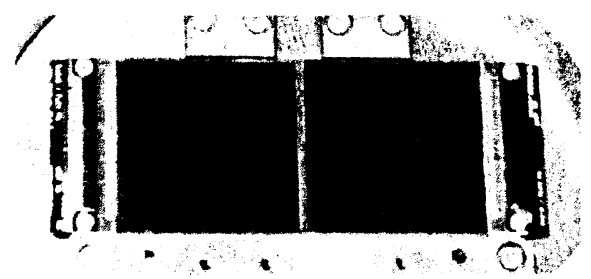

(b) Two HAWAII-2RG SCAs

Figure 4. The NIRSpec DS incorporates (a) two TIS SIDECAR ASICs and (b) two $\lambda_{\text {co }} \sim 5 \mu \mathrm{m}$ H2RG SCAs. These components are shown mounted in DCL ground support equipment (GSE). The flight mounts in NIRSpec are different.

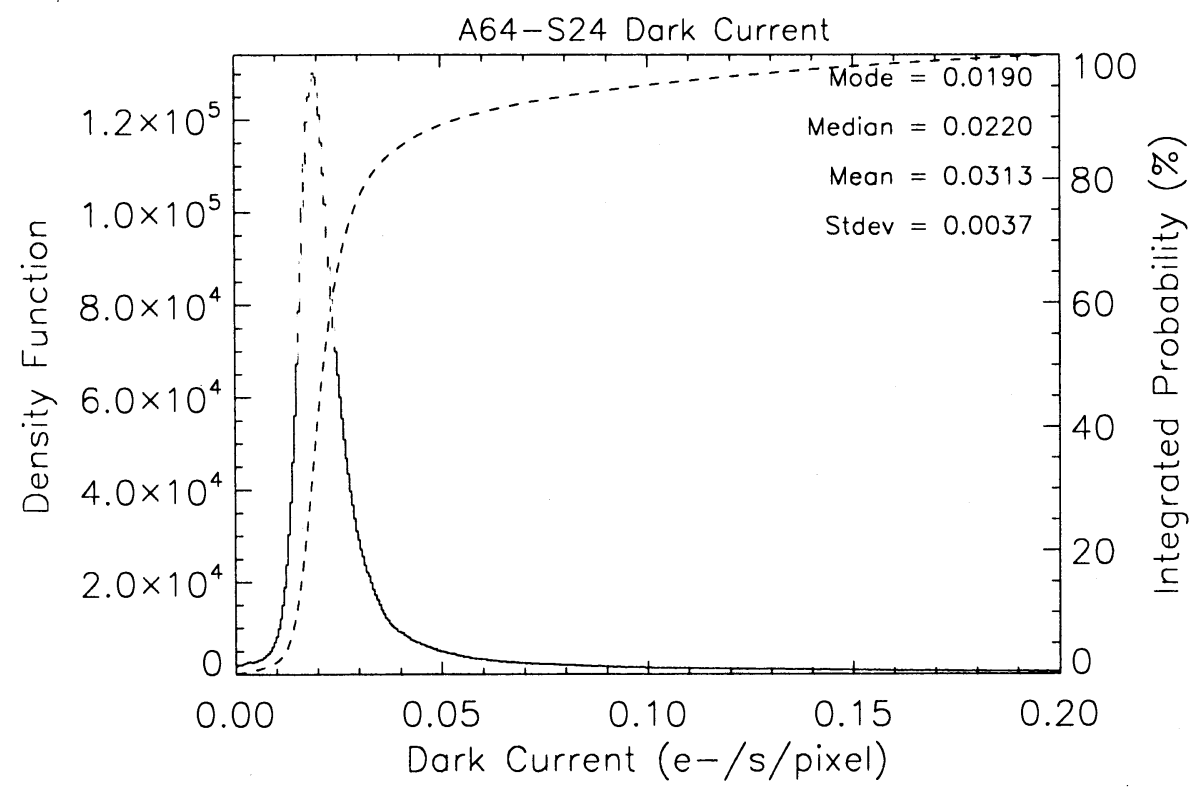

Figure 5. SIDECAR ASICs are being used to control H2RG SCAs in the DCL today. This figure shows the dark current histogram of engineering grade SCA H2RG-S024 driven by engineering grade SIDECAR A64. Both components are being operated at $\mathrm{T} \sim 37 \mathrm{~K}$. Although engineering grade parts like these do not meet flight specification, they are invaluable for validating test equipment and procedures.

The dark current histogram (Fig. 5), and total noise histograms (Fig. 6), are made from the same data set which contained 110 identical integrations. The readout mode was MULTI- $88 \times 1$, with 88 frames taken at a constant cadence of $t_{f}=10.62 \mathrm{~s}$ sampling-up-the-ramp. The detector reverse bias was $100 \mathrm{mV}$. We typically bias NIRSpec detectors somewhat higher, to $\sim 175 \mathrm{mV}$, to ensure meeting the $6 \times 10^{4} e^{-}$well depth requirement (Tab. 1). For this particular test, we were interested in exploring the low dark current limits of the engineering grade $\mathrm{SC} A$.

Using Equation 1, it can be shown that the read noise contribution to the total noise is about $\sigma_{\text {CDS }}=$ $26.6 e^{-}$rms. Here, $\sigma_{\text {CDS }}$ is the read noise per correlated double sample (CDS). Each CDS includes two reads of the detector, one at the beginning of the integration and one at the end. Early on in JWST, it was agreed that read noise per CDS would be the standard way of reporting noise because it allows for complete removal of the $k T C$ noise associate with resetting the integrating node of each pixel (see e.g. Ref. j). 

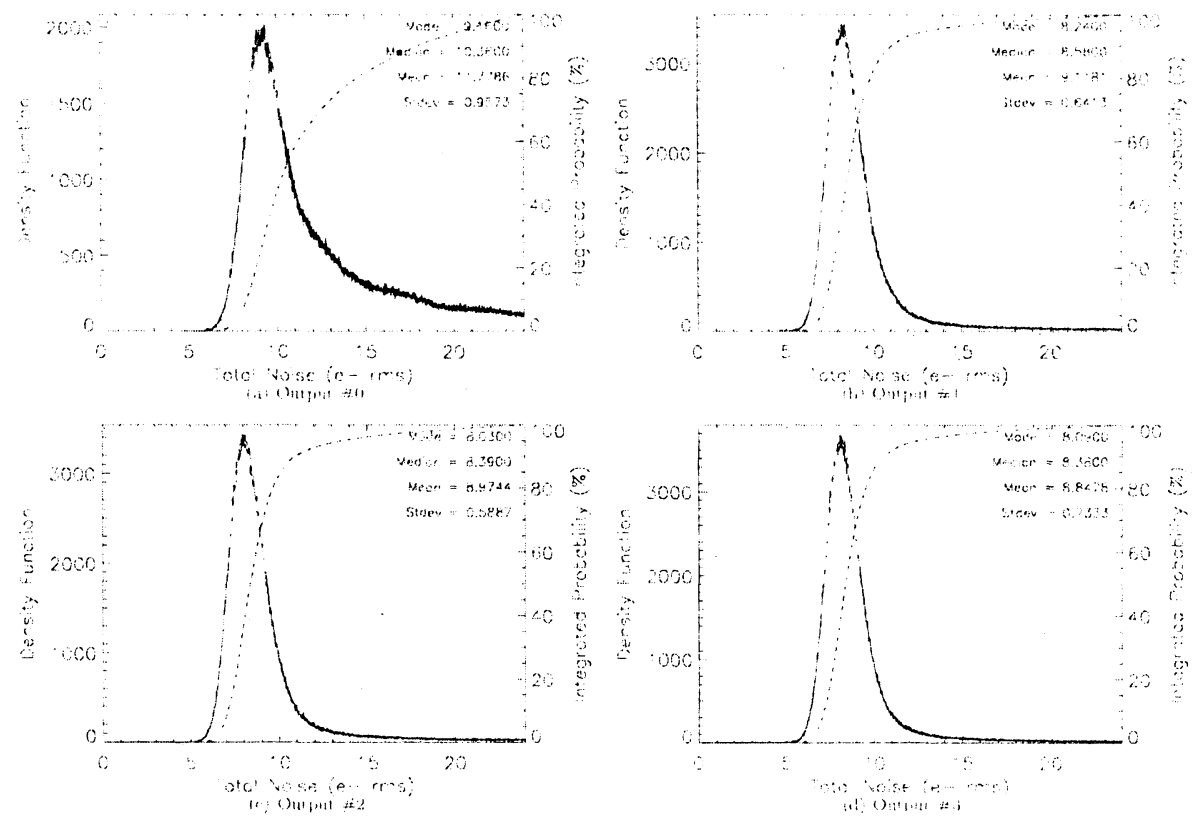

Figure 6. Total noise histograms for engineering grade SCA H2RG-S024 driven by engineering grade SIDECAR A64. The operating conditions are the same as in Figure 5.

\section{PLANNING FOR OPERATIONS: DETECTOR READOUT IN THE SPACE RADIATION ENVIRONMENT}

NIRSpec's detectors will be read out at a constant cadence using a generalize up-the-ramp sampled readout scheme that we have dubbed, "MULTIACCUM." Similar readout schemes have been used by other space instruments, including HST's NICMOS and Spitzer's IRAC. One advantage of up-the-ramp sampling for space missions is that cosmic ray hits can be detected in pixel ramps, and calibrated out with minimal data loss by retaining samples on either side of the hit. To conserve data volume, JWST averages "frames" of pixels into "groups" prior to saving data in the solid state recorder. The MULTIACCUM readout pattern and its parameters, as it is being implemented for JWST, is shown in Fig. 7.

For NIRSpec, the integration time, $t_{\text {int }}$, is the time between digitizing pixel $[0,0]$ in the first frame of the first group, and digitizing the same pixel in the first frame of the last group. The small overhead associated with finishing the last group is not included in the integration time.

Other important time intervals include the frame time, $t_{f}$, and the group time, $t_{g}$. The frame time is the time interval between reading pixel $[0,0]$ in one frame, and reading the same pixel in the next frame within the same group. The group time is the time interval between reading pixel $[0,0]$ in the first frame of one group, and reading the same pixel in the first frame of the next group. For NIRSpec, the integration time is related to the group time as follows, $t_{\text {int }}=(n-1) t_{g}$.

In order to model the noise in a MULTIACCUM sampled integration, correlations in the noise between frames and groups must be considered. Modeling these correlations are non-trivial, and for bright sources errors on the order of $10 \%$ can be expected if they are neglected. On the other hand, for an ultra-low dark current SCA tested under ultra-low background conditions, these correlations can be safely neglected and shot noise on integrated dark current can be added in quadrature with read noise. For a wide range of source brightnesses and backgrounds. Rauscher et al. (2007) show that a general expression for the total noise variance of an electronically. shuttered instrument using MULTIACCUM readout is, ${ }^{4}$

$$
\sigma_{\text {total }}^{2}=\frac{12(n-1)}{m n(n+1)} \sigma_{\text {rcad }}^{2}+\frac{6\left(n^{2}+1\right)}{5 n(n+1)}(n-1) t_{g} f-\frac{2(2 m-1)(n-1)}{m n(n+1)}(m-1) t_{f} f .
$$




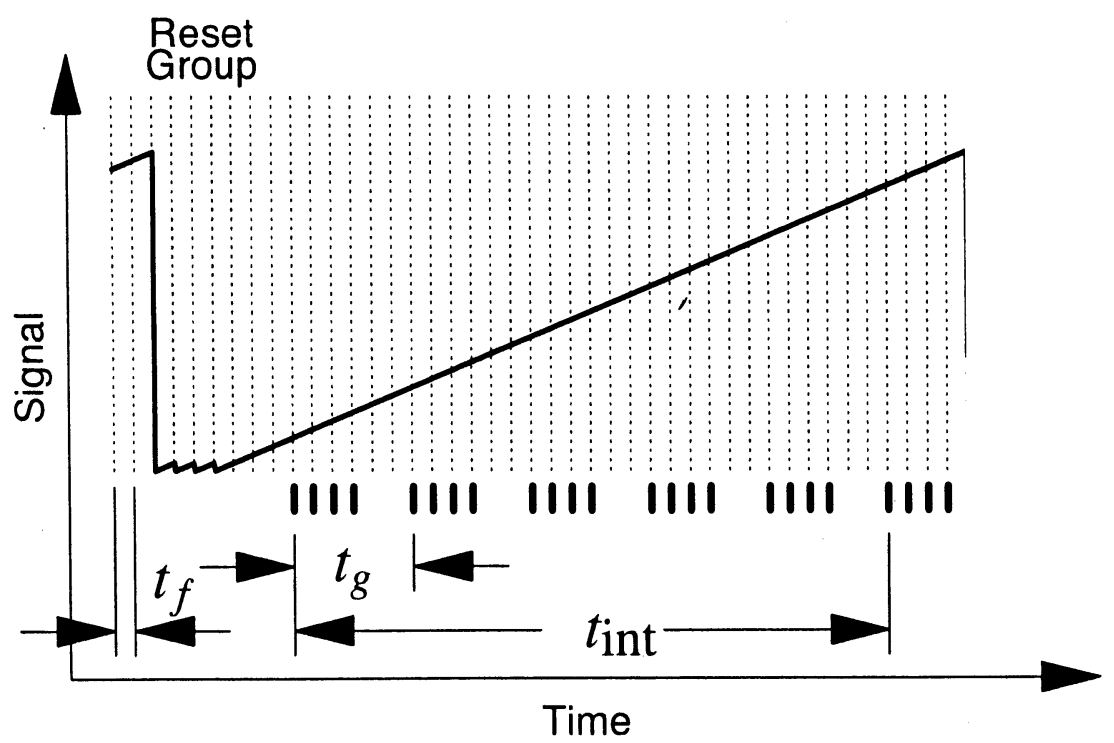

Figure 7. NIRSpec's detectors use MULTIACCUM sampling. The detector is read out at a constant cadence of one frame every $t_{f} \approx 10.5$ seconds. Although frames are clocked and digitized at a constant cadence, to conserve data volume, not all frames are saved. In this figure, saved frames are indicated by short, double width lines. Likewise, to conserve downlink bandwidth, not all frames are downlinked to the ground. Saved frames are co-added in the FPAP and averaged, resulting in one averaged group of data being saved to the solid state recorder every $t_{g}$ seconds. The resulting FITS file, consisting of a sampled-up-the-ramp data cube with points spaced at $t_{g}$ intervals, is downlinked to the ground for further processing.

Table 2. Model Parameters for Common Readout Modes

\begin{tabular}{lccl}
\hline \hline Readout Mode & $n$ & $m$ & Comments \\
\hline MULTI-22×4 & 22 & 4 & JWST NIRSpec baseline \\
MULTI-6 $\times 8$ & 6 & 8 & JWST NIRCam baseline \\
CDS & 2 & 1 & Correlated double sampling \\
MCDS- ${ }^{2}$ & 2 & 8 & Also known as Fowler-8 \\
\hline
\end{tabular}

${ }^{2}$ For MCDS readout modes, $t_{g}=t_{\text {int }}$.

In this expression, $\sigma_{\text {total }}$ is the total noise in units of $e^{-} \mathrm{rms}, \sigma_{\text {rcad }}$ is the read noise per frame in units of $e^{-}$rms, and $f$ is flux in units of $e^{-} \mathrm{s}^{-1}$ pixel $^{-1}$, where $f$ includes photonic current and dark current. The noise model includes read noise and shot noise on integrated flux, which is correlated across the multiple non-destructive reads sampling-up-the-ramp. For the special case of dark integrations, $f=i_{\text {dark }}$.

Equation 1 can also be used to model CDS and MCDS readout modes because both are special cases of MULTIACCUM. Table 2 summarizes the parameters to use for some common readout schemes. For more information on MULTIACCUM readout and noise modeling, the interested reader should see Ref. 4.

\section{SUMMARY}

This article provides a snapshot of the current developmental status of the JWST NIRSpec DS, provides early test results using TIS SIDECAR ASICs to drive H2RG SCAs at T $\sim 37 \mathrm{~K}$, and describes the planned MULTIACCUM readout mode and how this facilitates operation in the space radiation environment at L2. For more information on the JWST NIR SCA readout mode, noise model, and as-tested performance of engineering grade SCAs, the interested reader is referred to Ref. 4. Mott et al. (2007), in these conference proceedings, provides additional information on the GSFC DCL test facility, and also on the test performance of some engineering grade parts. 


\section{ACKNOWLEDGMENTS}

This work was supported by NASA and ESA as part of the James Webb Space Telescope Project. Ori Fox wishes to thank NASA's Graduate Student Researcher Program for a grant to the University of Virginia.

\section{REFERENCES}

1. D. Mott et al., "Characterization of the detector subsystem for near infrared spectrograph on the james webb space telescope," Proc SPIE, 2007. In press.

2. J. P. Gardner, "The james webb space telescope," Space Science Reviews 123(4), 2006.

3. G. Bagnasco et al., "The near-infrared spectrograph (nirspec) instrument on board the james webb space telescope: an instrument overview," Proc SPIE, 2007. In press.

4. B. J. Rauscher et al., "Detectors for the james webb space telescope near-infrared spectrograph i: Readout mode, noise model, and calibration considerations," PASP , 2007. In press.

5. G. Rieke, Detection of Light: From the Ultraviolet to the Submillimeter Second Edition, Cambridge University Press, 2003. 